between the experimental and control groups for each day of testing.

The only significant difference between the groups was found to be on Day 3 activity level $(t=2.69, \mathrm{df}=35, \mathrm{p}<.05)$. The mean activity level of the maternal separation group on Day 3 was 228, while that for the control group was 174. Thus, the interaction effect is apparently a function of Day 3 activity only. The dependent variable of activity level, then, does not converge with the defecation data. Interestingly enough, however, when the four groups in this study are collapsed into a maternal separation group and a control group, there can be seen a definite trend toward decreased emotional reactivity in the experimental group, with significant differences occurring on Day 3. On Day 2, the maternal separation groups were also more active on the average (205) than the controls (178), but the difference is not significant. When we look at the data in this way, the maternally deprived animals seem to be moving in the direction of being less emotional than the controls. This interpretation becomes all the more fascinating when we find that the quadrants traversed data reveal a significant main effect of maternal separation. The deprived animals traversed more quadrants $(\vec{X}=27.61)$ than the controls $(\vec{X}=19.95$; $F=12.67$, df $=1 / 68, p<.001)$. As these data are typically interpreted, the maternal separation groups were less emotional than the controls. A significant days effect was also found $(F=6.04, d f=2 / 136$, $\mathrm{p}<.005)$. The experimental group showed little change between Day I (28.8) and Day 3 (27.6), while the controls dropped in quadrants traversed from 23.8 on Day 1 to 17.3 on Day 3. Pearson product-moment correlation coefficients between activity level and quadrants traversed among the groups yielded high positive correlations ranging from .85 to .94.

Although the defecation data reflect increased emotionality for maternally deprived animals, previous studies have pointed out the weaknesses in using defecation as a measure of emotionality (e.g., LaBarba et al, 1968). It is interesting to note that nonisolated mice defecated more than isolated ones, and to speculate on the notion that perhaps defecation as a territorial response is related to early experiential variables and not entirely a matter of innate mechanisms.

It is suggested that the data obtained on activity level and quadrants traversed in this study offer some evidence to hypothesize that maternal deprivation, if severe enough, in intact litters of mice may decrease adult emotional reactivity, and that such reduction is a function of increased stimulation input occurring during infancy as a function of the maternal deprivation. It is further suggested that when littermates of rats and mice are subjected to a maternal separation paradigm, as reported here, increases in stimulation input to the infants result as a function of temperature reductions, quantitative and qualitative changes in maternal behavior, hunger, and increases in general activity and intralitter stimulation resulting from visceral stimulation. Such effects would be consistent with Denenberg's theory of infantile stimulation (1964). The studies dealing with maternal separation in rodents have obtained negative results perhaps because of the compensatory effects of increased littermate stimulation, etc., resulting from the maternal separation treatment. The present study, along with others reported here, may perhaps be more accurately described as the effects of littermate stimulation rather than maternal deprivation.

\section{REFERENCES}

DENENBERG, V. H. Critical periods, stimulus input, and emotional reactivity: A theory of infantile stimulation. Psychological Review, 1964, 71, 335-351.

HERSHER, L. Maternal deprivation in goats. Developmental Psychology, 1969, 1, 95-102.
IGEL, G. J., \& CALVIN, A. D. The development of affectional responses in infant dogs. Journal of Comparative \& Physiological Psychology, 1960, 53, 302-305.

LaBARBA, R. C., LUTZ, R., \& WHITE, J. Partial maternal separation and adult emotional reactivity in BALB/c mice. Psychonomic Science, 1968, 12, 301-302.

MASON, W. A. Early social deprivation in the non-human primate: Implications for human behavior. In D. C. Glass (Ed.), Environmental influences. New York: Rockefeller, 1968. Pp. 70-101.

NEWELL, T. G. Effects of maternal deprivation on later behavior in two inbred strains of mice. Psychonomic Science, 1967, 9, 119-120.

SEITZ, P. F. D. The effects of infantile experience upon adult behavior in animal subjects: I. Effects of litter size during infancy upon adult behavior in the rat. American Journal of Psychiatry, 1954, 110, 916-927.

THOMAN, E. B., \& ARNOLD, w. J. Effects of incubator rearing with social deprivation on maternal behavior in rats. Journal of Comparative \& Physiological Psychology, 1968a, 65, 441-446.

THOMAN, E. B., \& ARNOLD, W. J. Incubator rearing of infant rats without the mother: Effects on adult emotionality and learning. Developmental Psychology, 1968b, 1, 219-222.

NOTE

1. This study is based on a Master's thesis submitted by the second author to the Department of Psychology, University of South Florida, in partial fulfillment of the requirements for the MA degree.

\title{
Paradoxical sleep in the rat: Comparison of early and late blinding
}

A. MICHAEL ANCH ${ }^{2}$ and DONALD I. TEPAS, St. Louis University, St. Louis, Mo. 63103

Electrophysiological recordings of sleep in adult rats blinded prior to weaning were significantly different from those recorded in littermates blinded after weaning. Behavior on an open-field test failed to indicate any significant differences between these two groups. Thus, it appears that an early reduction of external sensory input can modify brain activity during sleep.

The functional significance of paradoxical sleep (PS), frequently termed rapid-eye-movement sleep (REM), is not clearly understood. Roffwarg, Munzio, \& Dement (1966) suggest that it is required for the normal development of the central nervous system (CNS). They hypothesize that a reduction of exogenous inputs to the CNS will result in an increase of endogenous activity such as that associated with paradoxical sleep.

In our experiment, we attempted to investigate the interaction between neural feedback and sensory stimulation. We predicted that a reduction of external sensory input to the CNS of rats early in life would result in an increase of PS activity of adult rats. Specifically, the purpose of this study was to determine the effects of early vs late blinding on paradoxical sleep. We compared these measures with the behavior of the two groups with respect to ambulatory activity in an open-field apparatus.

\section{APPARATUS AND PROCEDURE}

Twenty-one (Wistar strain) rats, the result of matings in our own laboratory, were assigned randomly, using a split-litter 
technique, to an early- or late-blinded group. Ten animals in the late-blinded group and 11 in the early-blinded group completed all testing. Early blinding was defined as gross cauterization of the developing optic regions at exactly 5 days after birth. Late blinding was defined as total removal of the eyeballs (with severing of the optic nerve) at approximately 80 days of age.

All animals completed their weaning period by 18 days of age and were separated from their mothers at this time. We raised all the animals in the same deprived environment, each rat being assigned randomly to an individual stovepipe cage.

We removed the Ss from their stovepipe cages at 120 to 170 days and conducted behavioral tests and electrophysiological recordings. Using a similar procedure and the same apparatus as Lamp (1966), we measured the amount of ambulatory activity in an open-field test immediately after removal from the stovepipe cages. We began the electrophysiological recordings, defined as the amount of PS activity in a 2-h sample, from 1 to 14 days later. A Grass Model IV polygraph with a Model 7 P5 A EEG preamplifier was used for recording EEG and EMG. We attached three special silver disk electrodes ( .25 -in. diam) to the scalp of the animal. In addition, we attached an ear-clip reference electrode to the ear and inserted a subdermal platinum alloy needle (approximately $.25 \mathrm{in}$. long) into neck muscle. All electrodes were held firmly in place by collodion, administering Fluothane anesthesia to perform the electrode application.

To minimize the possible confounding effects of anesthesia on the electrophysiological recording, we waited approximately $2 \mathrm{~h}$ after placing the animal in the electrophysiological recording booth before collecting data. We divided the first $120 \mathrm{~min}$ after sleep onset into 1 -min intervals and analyzed them according to a modification of the criteria established by McGinty (1967).

\section{RESULTS AND DISCUSSION}

The relevant variables measured in the electrophysiological recording were percentage of PS and the latency to the first PS period. We analyzed the records of all 21 rats, using the Mann-Whitney $U$ test. The results of this test for the comparison of the effects of early- vs late-blinded rats on the amount of PS recording for a $2-\mathrm{h}$ criterion period showed that the amount of PS was significantly different between the two groups. The early-blinded rats showed significantly more PS than the late-blinded rats for the 2 -h sample $(U=23, p<.025)$. Figure 1 compares the mean amount of PS



Fig. 1. Comparison of the mean amounts of paradoxical sleep in early and late blinded rats.

in the two groups. No sex differences were evident in the data.

We also used the Mann-Whitney $U$ test in analyzing the results of latency to the first PS period. The criterion used for latency to first PS was the occurrence of a PS period of at least $1-\mathrm{min}$ duration. The results were in the expected direction. The early-blinded rats showed a shorter latency to first PS than did the late-blinded rats $(\mathrm{U}=27, \mathrm{p}<.025)$. Figure 2 compares the mean latency to first PS for the two groups. We also compared the data for the same 21 animals on the open-field test. We recorded the behavioral data in 2-min blocks for a total period of $8 \mathrm{~min}$. We compared total ambulatory activity at each 2-min interval and statistically analyzed them for the two groups. A 2 by 4 analysis of variance showed only significant effects for the variable of time of testing: amount of ambulatory activity measured at the end of $2,4,6$, and $8 \mathrm{~min}$. There were no significant effects for early-vs late-blinded rats on the amount of ambulatory activity.

The comparison of the electrophysiological recordings and the behavioral test data indicates that there were significant differences in the PS data only. The lack of a significant difference between the two groups on the behavioral measure is noteworthy. The fact that earlyand late-blinded rats showed no differences in ambulatory activity suggests that the early-blinded rats were behaviorally no more active than the late-blinded rats. These results are in agreement with an earlier study by Hymovitch (1952).

The supposition is that paradoxical sleep acts as a substitute for exogenous stimulation. This "substitution effect," then, would account for the lack of

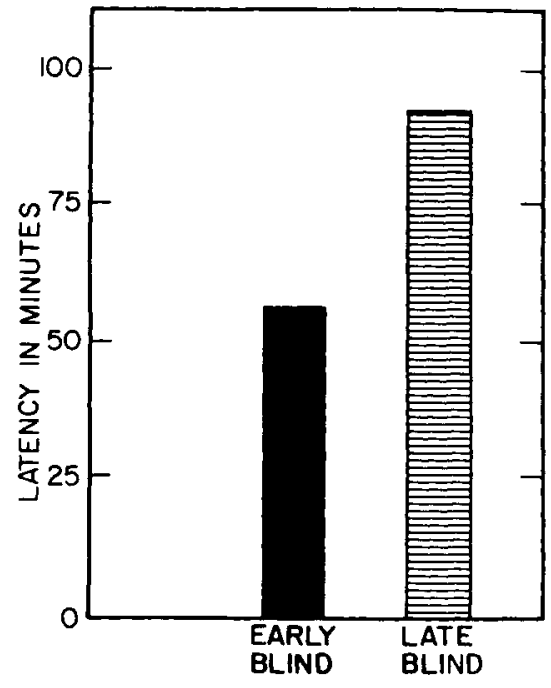

Fig. 2. Comparison of the mean latencies to first PS in early and late blinded rats.

significant differences in the effects of the behavioral measure between the two groups. The fact that there were significant differences in the electrophysiological recordings in the direction of increased PS for the early-blinded rats, suggests an interaction between neural feedback and sensory stimulation for sensory-deprived rats, such that the reduction of external sensory input to the CNS will result in increased PS activity. More specifically, the study suggests a feedback system in which early-blinded rats showed more of a compensatory mechanism of endogenous activity in the form of PS activity than the late-blinded rats. These results support the theoretical notions of Roffwarg et al (1966) concerning the functional significance of paradoxical sleep.

The results suggest that additional developmental studies are warranted to evaluate the functional significance of paradoxical sleep.

\section{REFERENCES}

HYMOVITCH, B. Effects of environmental variation of problem-solving in rats. Journal of Comparative \& Physiological Psychology, $1952,45,313-321$

LAMP, R. E. Offspring effects of mother anxiety. Unpublished doctoral dissertation, Washington University, St. Louis, 1966.

McGINTY, D. J. Neural control of sleep patterns in the rat. University Microfilms, Ann Arbor, Michigan, 1967.

ROFFWARG, H. P., MUNZIO, J.N., \& DEMENT, W. C. Ontogenetic development of the human sleep-dream cycle. Science, 1966, 152, 604-617.

\section{NOTES}

1. This paper was presented at the 1969 Psychonomic Society Meeting in St. Louis.

2. Reprint requests should be sent to $A$. Michael Anch, Department of Psychology, St. Louis University, 221 No. Grand Blvd., St. Louis, Mo. 63103. 